\title{
ANALYSES OF RESIDUAL THERMAL STRESSES IN CERAMIC MATRIX COMPOSITES
}

C. H. Hsueh and P. F. Becher, Metals and Ceramics Division, Oak Ridge National Laboratory, P. O. Box 2008, Oak Ridge, Tennessee 37831-6068

Residual thermal stresses in ceramic matrix composites containing either ellipsoldal $\mathbf{S}$ I I inclusions or short fibers (i.e., fibers of finite length) are considered. First, the residual stresses in ellipsoidal inclusions are uniform, and they are analyzed using a modified Eshelby model. Although closed-form analytical solutions are obtained, their formulations are formidable. When the aspect ratio of the ellipsoid is 0,1 , or infinity, simple analytical solutions can be obtained using different models, and they are in excellent agreement with those obtained from the modified Eshelby model. Second, residual stresses in short fibers are nonuniform, and they are analyzed using a modified shear lag model, in which imaginary fibers are introduced to satisfy the continuity condition at the fiber ends. The analytical solutions are compared to the experimental results.

\section{INTRODUCTION}

The mechanical properties of ceramics are intimately related to their microstructures. The brittleness of ceramics can be reduced by incorporating dispersed inclusions such as second phases, fibers, or whiskers [1-3]. Such ceramic composites are fabricated at elevated temperatures. As they are cooled to the room temperature, residual thermal stresses develop due to the thermomechanical mismatch between the matrix and the inclusions. Accordingly, these residual thermal stresses must be considered in the material design and various applications.

The purpose of the present study is to analyze the residual thermal stresses in ceramic composites containing either ellipsoidal inclusions or short fibers. When the inclusions are ellipsoidal, closed-form analytical solutions for the residual stresses can be obtained using a modified Eshelby model [4,5]; however, their formulations are formidable. For the special cases of disc-shaped, spherical, or fiber-shaped inclusions (i.e., the aspect ratio of the ellipsoid is 0,1 , or infinity), the residual stresses can be analyzed using simplified models, which provide simple analytical solutions. The analytical solutions are then compared to both experimental [6] and finite element [7] results, and effects of the aspect ratio and the volume fraction of inclusions on residual thermal stresses are examined. When the inclusions are short fibers, closed-form analytical solutions for the residual stresses can be obtained using a modified shear lag model [8]. To satisfy the continuity condition at the fiber ends, a technique of assuming imaginary fibers in the matrix is adopted. The predicted axial strain along the fiber length is then compared to the existing experimental measurements [9]. 


\section{ELLIPSOIDAL INCLUSIONS}

The residual thermal stresses in an ellipsoidal inclusion embedded in an infinite matrix were analyzed in the Eshelby model [4], which was subsequently modified to account for a finite volume fraction of inclusions [5]. The results showed that residual thermal stresses in ellipsoidal inclusions are uniform and are functions of the thermomechanical properties of the constituents of the composite, the temperature change for stress development, and the aspect ratio and the volume fraction of inclusions. This modified Eshelby model has been adopted by $\mathrm{Li}$ and Bradt [10] and by Hsueh and Becher [11] to calculate residual thermal stresses in whisker-reinforced ceramic composites, in which whiskers were simulated as ellipsoidal inclusions. The ceramic matrix is polycrystalline and is isotropic in both elastic constants and the coefficient of thermal expansion (CTE). The inclusions can have preferred orientations which, in turn, can result in anisotropies in both elastic constants and the CTE. These anisotropies were considered by $\mathrm{Li}$ and Bradt, and the solutions for the residual thermal stresses were formulated in matrix forms. Hsueh and Becher considered anisotropy in the CTE only for the inclusions, and closed-form analytical solutions were obtained for the residual thermal stresses. It was found that compared with the anisotropy in the CTE, the anisotropy in elastic constants has a minor effect on the residual thermal stresses [11]. Although solutions for the residual thermal stresses in the ellipsoidal inclusion obtained from the modified Eshelby model are closed-form, their formulations are formidable. However, when the ellipsoidal inclusion has a special shape such as disc-shaped, spherical, or fiber-shaped, simple analytical solutions can be obtained using different models and they are summarized as follows.

Disc-Shaped, Spherical, and Fiber-Shaped Inclusions

Using Cartesian coordinates, $X_{1}, X_{2}$, and $X_{3}$, with the $X_{3}$-axis as the axis of symmetry for the ellipsoidal inclusion, the inclusion has a radius $a$ in the $X_{1}$ and the $X_{2}$ directions and a radius $c$ in the $X_{3}$ direction and its aspect ratio is $c / a$. When $c / a$ approaches zero, the inclusion becomes a disc with radius $a$ and thickness $c$ and $a>>c$. The representative volume element of the composite is a disc-shaped inclusion sandwiched by two discs of matrix (Fig. 1a). In the $X_{3}$ direction, there is no constraint and the residual thermal stresses are zero in both the matrix and the inclusion (i.e., $\sigma_{3}=0$ ). The solution for the residual thermal stress in the inclusion in the $X_{1}$ (and the $X_{2}$ ) direction, $\sigma_{1}$, can be obtain from conditions of (i) continuity of the displacement between the inclusion and the matrix in the $X_{1}$ (and the $X_{2}$ ) direction, and (ii) mechanical equilibrium, such that [11]

$$
\sigma_{1}=\left(\alpha_{\mathrm{m}}-\alpha_{1}\right) \Delta T /\left[\left(1-v_{\mathrm{e}}\right) / E_{\mathrm{e}}+f\left(1-v_{\mathrm{m}}\right) /(1-f) E_{\mathrm{m}}\right]
$$

where $E$ is Young's modulus, $v$ is Poisson's ratio, the subscripts e and $\mathrm{m}$ denote the ellipsoidal inclusion and the matrix, respectively, $f$ is the volume fraction of the inclusion, $\alpha_{1}$ is the CTE of the inclusion in the $X_{1}$ (and the $X_{2}$ ) direction, $\alpha_{\mathrm{m}}$ is 
the isotropic CTE of the matrix, and $\Delta T$ is the temperature change for stress development.
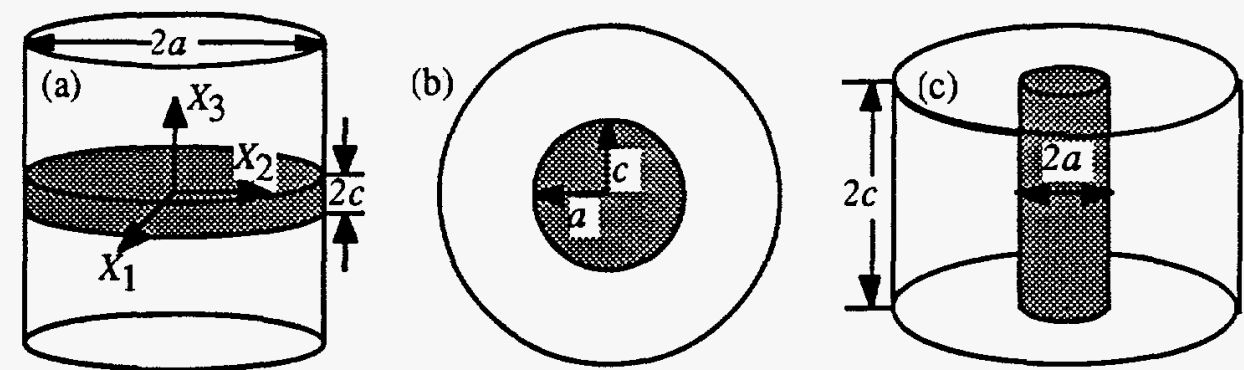

Fig. 1. Schematic showing the representative volume element for composites containing (a) disc-shaped ( $c / a<<1)$, (b) spherical ( $c / a=1)$, and (c) fiber-shaped $(c / a>1)$ inclusions.

When c/a equals to one, the inclusion becomes a sphere with a radius $a$. The representative volume element of the composite is a composite sphere (Fig. 1b). This problem has been analyzed when the CTE in the inclusion, $\alpha_{e}$, is isotropic. In this case, the thermal stress in the spherical inclusion is hydrostatic, and the solution has been given in Ref. [12]. When comparison with the solution obtained from the modified Eshelby model for the case of $c / a=1$ is made, $\alpha_{e}=\left(2 \alpha_{1}+\alpha_{3}\right) / 3$ is adopted where $\alpha_{3}$ is the CTE of the inclusion in the $X_{3}$ direction.

When $c / a$ is much greater than one, the inclusion becomes an infinitely long fiber with a radius $a$. The representative volume element of the composite is a composite cylinder (Fig. 1c). The residual thermal stress in the fiber has been analyzed, and the analytical solutions are given in Ref. [13].

\section{Comparison}

The residual thermal stresses in two composites, $\mathrm{SiC}$ whisker-reinforced $\mathrm{Al}_{2} \mathrm{O}_{3}$ [6] and graphite fiber-reinforced aluminum [7], are considered. It is noted that the graphite/Al composite is a metal matrix composite. However, its elastic residual thermal stresses have been calculated using a finite element method [7], and they can be used to compare with the present analytical solutions.

$\mathrm{SiC}$ whisker-reinforced $\mathrm{Al}_{2} \mathrm{O}_{3}$ composites: Residual thermal stresses have been measured for a hot-pressed $\alpha-\mathrm{Al}_{2} \mathrm{O}_{3}$ matrix containing different volume fractions of $\beta$-SiC whiskers using $\mathrm{X}$-ray diffraction [6]. It has been reported that the measured average residual thermal stresses, $\bar{\sigma}$, in the inclusion agree well with those predicted from the modified Eshelby model [6]. It is noted that elastic constants of the single-crystal $\mathrm{SiC}$ whisker are highly anisotropic [10]. Adopting the average elastic property for $\mathrm{SiC}$ whiskers, the calculated magnitude of $\bar{\sigma}$ by Hsueh and Becher [11] is only $\sim 5 \%$ lower than that predicted in Li and Bradt's analysis, in which anisotropy in elastic constants is considered. 
Graphite fiber-reinforced Al composites: Elastic residual thermal stresses have been calculated for a continuous unidirectional Pitch 55 graphite fiber-reinforced A201 aluminum composites using a finite element method [7]. Two types of fiber arrays, square and hexagonal, were adopted in the finite element modeling [7]. The material properties are listed in Table I. The CTE of the graphite fiber is highly anisotropic. Using the finite element method, the residual thermal stresses per each ${ }^{\circ} \mathrm{C}$ increase in the temperature were calculated for various fiber volume fractions. The calculated radial stress is not uniform around the interface due to the interaction between fibers, its variation around the interface becomes greater as the volume fraction of fibers increases, and the maximum radial stress at the interface is considered in the finite element results [7].

Table I. Material Properties of Graphite Fiber-Reinforced Aluminum Composites

\begin{tabular}{|l|l|l|l|l|}
\hline & $E(\mathrm{GPa})$ & $v$ & $\alpha_{1}\left(\times 10^{-6} /{ }^{\circ} \mathrm{C}\right)$ & $\alpha_{3}\left(\times 10^{-6} / \mathrm{O}^{\circ}\right)$ \\
\hline graphite & 385 & 0.2 & 25 & 0 \\
\hline aluminum & 70 & 0.35 & 23.6 & 23.6 \\
\hline
\end{tabular}

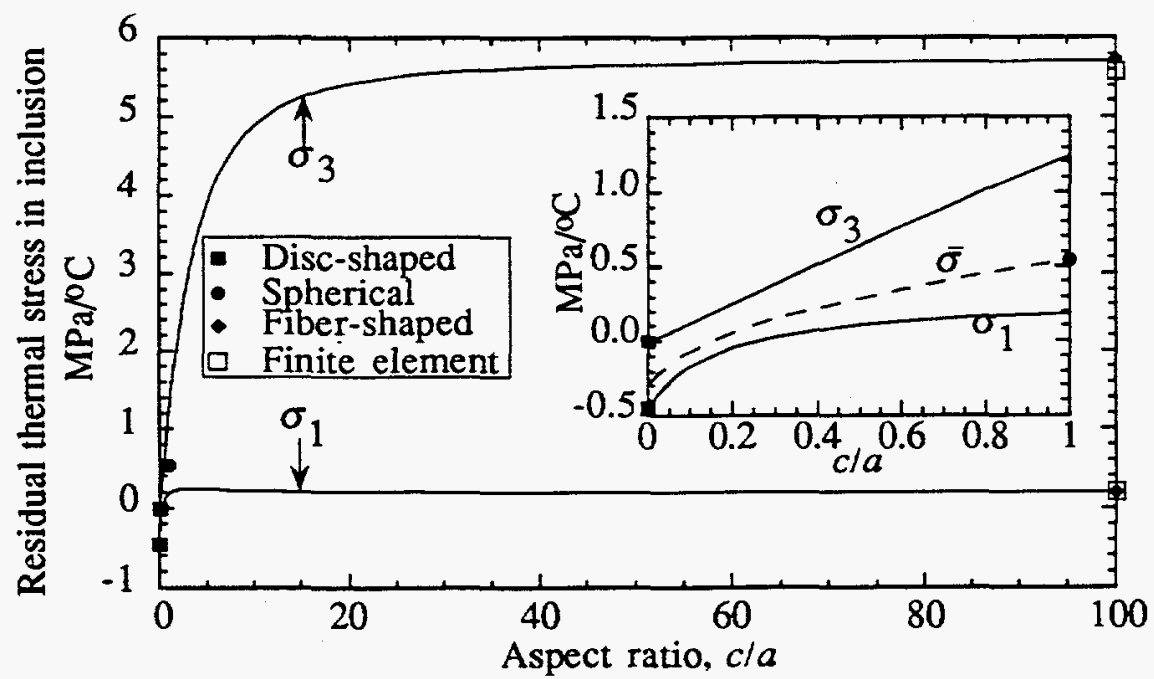

Fig. 2. The residual thermal stresses, $\sigma 1$ and $\sigma_{3}$, in ellipsoidal inclusions per ${ }^{\circ} \mathrm{C}$ increase in temperature calculated using the modified Eshelby model as functions of the aspect ratio of inclusions, $c / a$, for $10 \mathrm{vol} \%$ graphite/Al composites. The analytical solutions for disc-shaped, spherical, and fiber-shaped inclusions obtained from different models as well as finite element results are also shown.

Using the given material properties in Table $I$, the stresses, $\sigma_{1}$ and $\sigma_{3}$, in the ellipsoidal inclusion per ${ }^{\circ} \mathrm{C}$ increase in the temperature are calculated as a function of the aspect ratio, $c / a$, for $f=0.1$ in Fig. 2. The calculated results from the three analytical solutions for disc-shaped, spherical, and fiber-shaped inclusions are also shown. For $f=0.1$, the finite element results are not sensitive to the fiber arrays, and only one set of finite element results for $\sigma_{1}$ and $\sigma_{3}$ is shown in Fig. 2. Detailed comparison between the results from modified Eshelby model and those 
from analytical solutions for disc-shaped and spherical inclusions is shown in the insert of Fig. 2. It is noted that the anisotropy in CTE of the inclusion is not considered in the composite sphere model [12]. Hence, the residual hydrostatic stress, $\bar{\sigma}$, in the inclusion calculated from the modified Eshelby model is also shown in the inserted figure to compare with that obtained for the composite sphere model. Excellent agreement is obtained in Fig. 2. The magnitude of 03 increases from zero but the magnitude of $\sigma 1$ decreases from a finite value as $c / a$ increases from zero, and both $\sigma_{3}$ and $\sigma_{1}$ reach their asymptotic values when $c / a$ is sufficiently large.

The maximum interfacial radial stress, $\sigma$, and the axial stress in the matrix, $\sigma_{3}(\mathrm{~m})$, have been calculated using the finite element method for various fiber volume fractions, $f$. Since the finite element results were obtained for continuous fibers, the asymptotic values of $\sigma_{1}$ and $\sigma_{3}(\mathrm{~m})$ obtained from the modified Eshelby model are shown as functions of $f$ in Fig. 3 to compare with the finite element results. Excellent agreement between the analytical solutions and the finite element results is obtained when $f$ is less than 0.3 . The difference between the analytical and the finite element results at high volume fractions of fibers (e.g. $f>0.3$ ) is due to fiber-fiber interaction, which is considered in finite element solutions but not in analytical solutions. For finite element results, the square array of fibers shows a greater fiber-fiber interaction than the hexagonal array of fibers (Fig. 3) [7].

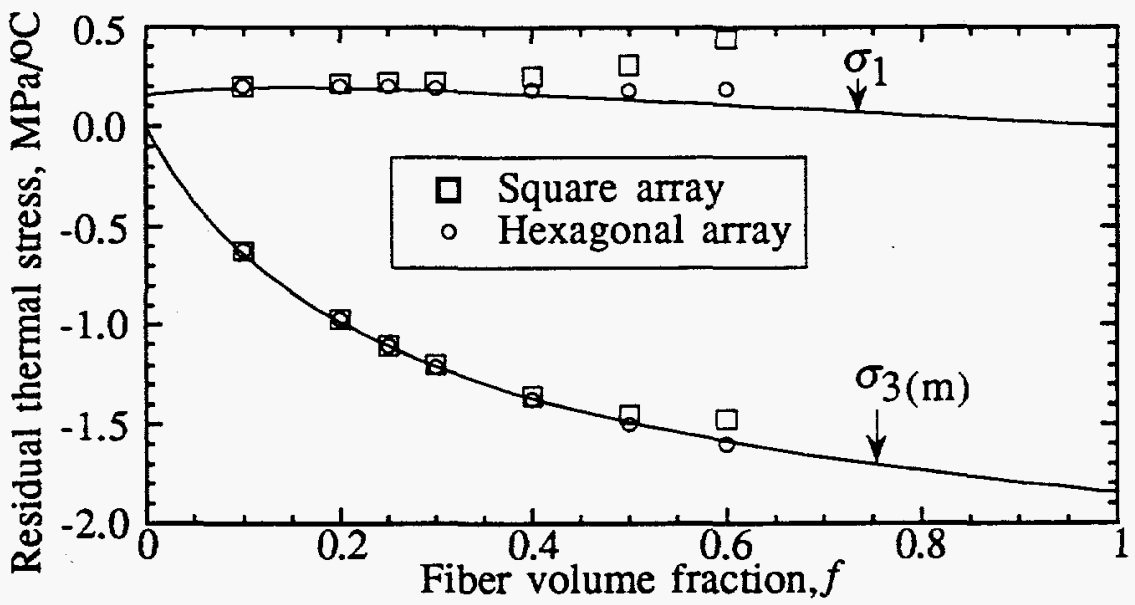

Fig. 3. The calculated asymptotic values of $\sigma_{1}$ and $\sigma_{3}(\mathrm{~m})$ per ${ }^{\circ} \mathrm{C}$ increase in temperature for graphite/Al composites. The finite element results for square and hexagonal arrays of fibers are also shown.

\section{SHORT FIBERS}

To obtain analytical solutions for the stress distribution in fiber-reinforced composites, the shear lag model [14] has been used extensively; however, the stresses at the fiber ends need to be predetermined in the analysis. For a fully 
embedded fiber, the fiber ends are stress-free if they debond from the matrix.

When the fiber ends are bonded to the matrix, stress transfer occurs at the bonded ends, and the stresses at bonded ends are finite. However, these finite stresses are not predetermined values. To define the boundary condition at the bonded ends, a technique of adding imaginary fibers to the shear lag model was developed recently [8]. While detailed analyses for the above problem can be found elsewhere [15], the procedures in deriving solutions are summarized as follows.

\section{Analyses}

The shear lag model for the representative volume element of composites containing short fibers is shown in Fig. 4. A fiber with a radius $a$ and a length $2 l$ is embedded at the center of a coaxial cylindrical matrix with a radius $b$ and a length $2 l$. The cylindrical coordinates, $r, \theta$, and $z$, are used. The fiber is bonded to the matrix at both the interface (i.e., at $r=a$ ) and the ends (i.e., at $z= \pm l$ ). During cooling of the composite from its fabrication temperature, thermal strains are induced in both the fiber and the matrix. An isotropic thermal expansion coefficient, $\alpha_{\mathrm{m}}$, is considered for the matrix. Transversely isotropic thermal expansion coefficients, $\alpha_{\mathrm{r}}$ in the radial and the tangential directions and $\alpha_{\mathrm{Z}}$ in the axial direction, are considered for the fiber. Due to the thermomechanical mismatch between the fiber and the matrix, residual stresses are induced, and stress transfer occurs between the fiber and the matrix through both the interface and the fiber ends.

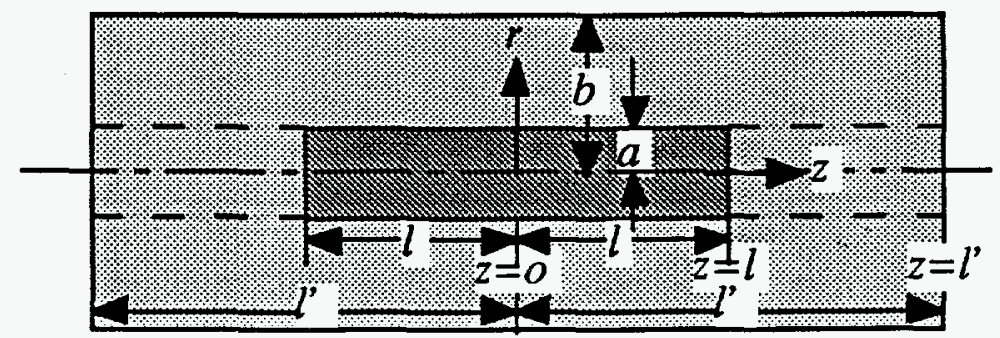

Fig. 4. Schematic showing the shear lag model for analyzing the residual thermal stresses in composites containing short fibers. The region between dashed-lines shows the imaginary fibers added to define the continuity condition at the ends of the real fiber.

The geometry described in Fig. 4 is symmetric, and only the region $z \geq 0$ is discussed. Stress transfer between the fiber and the matrix in the region $0 \leq z \leq l$ can be analyzed using a shear lag model; however, two questions remain. The first question regards whether stress transfer occurs in the region $l \leq z \leq l '$, in which there are no fibers and only the matrix exists. The second question regards the stress at the fiber end (i.e., at $z=l$ ) which is required as a boundary condition in solving the stress transfer problem; however, this stress is not a predetermined value. To address the above two questions, the technique of assuming imaginary fibers in the matrix is developed. The region between the fiber ends and the matrix surface (see the region between dashed-lines in Fig. 4) is treated as two imaginary fibers, which 
have the matrix properties. With the imaginary fiber, stress transfer in the region $l \leq z \leq l \mid$ can also be analyzed using a shear lag model provided that the stress in the imaginary fiber at $z=l$ is predetermined. The matrix has a free surface at $z=l$. Unless the stress at $z=l$ is zero, stress transfer in the region $l \leq z \leq l$ ' should occur. Due to the discontinuity of material properties at $z=l$, the stress at $z=l$ is expected to be different from zero. Hence, stress transfer occurs in both regions, $0 \leq z \leq l$ and $l \leq z \leq l$, and one shear lag model is required for each region to analyze stresses. However, due to the unknown stress at $z=l$, the solution of stress distribution within each region contains one unknown parameter. It is noted that the two regions join at $z=l$. Hence, both the axial stress and the shear stress along the interface obtained from the above two shear lag models for the above two regions should be continuous at $z=l$. These two continuity conditions at $z=l$ provide solutions for the two unknown parameters, and the solution for stress transfer in the entire composite is complete.

\section{Comparison}

The stress-induced axial strain along the length of a fully embedded fiber has been measured using fluorescence spectroscopy [9]. A model composite was fabricated by embedding a single PRD-166 alumina-zirconia fiber in a glass matrix, for which either Pyrex or soda-lime silicate (SLS) was used. The material properties are listed in Table II [9]. The fiber has a radius, $a$, of $10 \mu \mathrm{m}$, and a length, $2 l$, of $1.2 \mathrm{~mm}$ and $1.4 \mathrm{~mm}$, respectively, for PRD-166/Pyrex and PRD-166/SLS composites. The matrix radius is about 100 times the fiber radius (i.e., $b / a=100$ ), and the matrix length is about three times the fiber length (i.e., $\left.l^{\prime}=3 l\right)$. Also, since the measurements were performed at $\sim 22^{\circ} \mathrm{C}, \Delta T$ is taken as the difference between $22^{\circ} \mathrm{C}$ and the softening point (see Table II) of the glass matrix.

Table II. Material Properties of PRD-166 fiber, Pyrex and SLS [9]

\begin{tabular}{|l|l|l|l|}
\hline Property & PRD-166 & Pyrex & SLS \\
\hline Young's modulus (GPa) & 380 & 61 & 74 \\
\hline Poisson's ratio & 0.27 & 0.3 & 0.3 \\
\hline Softening Point $\left({ }^{\circ} \mathrm{C}\right)$ & - & 820 & 700 \\
\hline Thermal Expansion Coefficient $\left(\times 10^{-6}\right)$ & 9 & $3.2(3.85)^{*}$ & $9.2(10.9)^{*}$ \\
\hline
\end{tabular}

* Adjust for crystallization shrinkage.

The measured stress-induced axial strains along the fiber length are shown in Figs. $5 a$ and $b$ for PRD-166/Pyrex and PRD-166/SLS composites, respectively. The strain has a finite value at the fiber end, and its magnitude increases and reaches an asymptote in the central region. The positive and the negative signs for the strain correspond to tension and compression, respectively. It is noted that crystallization occurs in the glass matrix during processing and results in a volume shrinkage which, in turn, imposes a compressive stress on the embedded fiber [9]. To account for the shrinkage of the matrix due to crystallization, $\alpha_{\mathrm{m}}$ is increased in the present study to match the predicted to the measured asymptotic values of the 
stress-induced strain. Based on this modification of $\alpha_{\mathrm{m}}$, the predicted stressinduced axial strains along the fiber axis are shown in Fig. 5, and good agreement between the predicted and the measured results is obtained. The predicted stressinduced axial strains along the imaginary fiber (i.e., in the matrix) are also shown in Fig. 5; however, the experimental measurements of matrix strains are not available for comparison. Compared to the fiber, the matrix has a greater stress-induced axial strain around the fiber end. This is due to the lower Young's modulus of the matrix compared to the fiber.
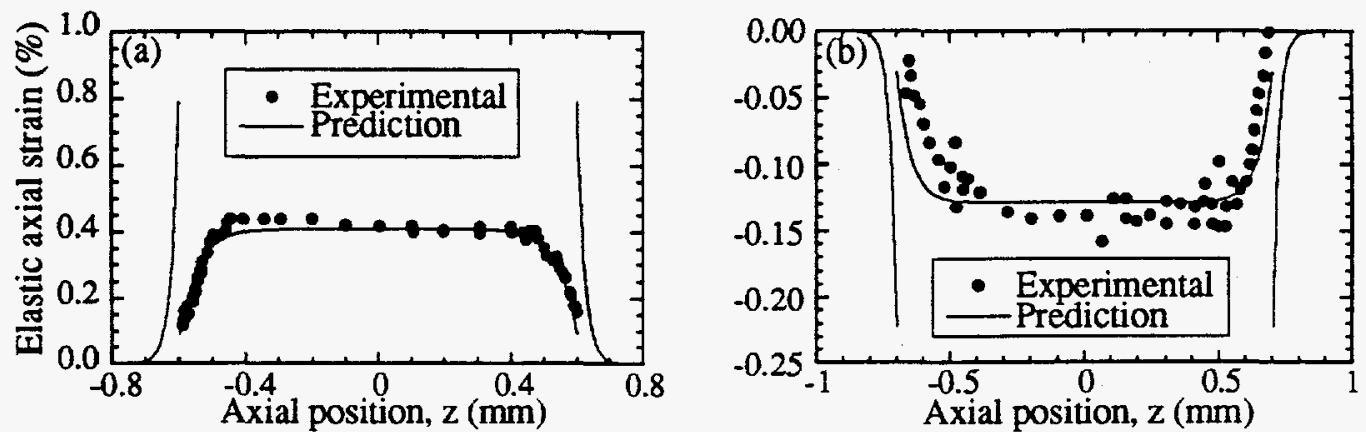

Fig. 5. Distributions of the stress-induced elastic axial strain along the fiber length for (a) PRD-166/Pyrex and (b) PRD-166/SLS composites. The predicted elastic axial strain in the imaginary fiber (i.e., the matrix) is also shown.

\section{CONCLUSIONS}

Residual thermal stresses in ceramic matrix composites containing either ellipsoidal inclusions or short fibers (i.e., fibers of finite length) are considered. First, the residual stresses in ellipsoidal inclusions are uniform, and they are analyzed using a modified Eshelby model. Closed-form analytical solutions are obtained; however, their formulations are formidable. When the aspect ratio of the ellipsoid is 0,1 , or infinity, simple analytical solutions can be obtained using different models, and they are in excellent agreement with those obtained from the modified Eshelby model. Excellent agreement is also obtained between the analytical solutions and the finite element results for graphite/Al composites when volume fraction of inclusions is less than 0.3 . The difference between the analytical and the finite element results at high volume fractions of fibers is due to fiber-fiber interaction, which is considered in finite element solutions but not in analytical solutions. Second, residual stresses in short fibers are nonuniform, and they are analyzed using a modified shear lag model. To satisfy the continuity condition at the fiber ends, a technique of assuming imaginary fibers in the composite is adopted. The predicted stress-induced axial strain along the fiber length is in excellent agreement with the existing experimental measurements. Along the fiber length, the predicted stress-induced axial strain has a finite value at the fiber ends, it increases with the distance from the fiber ends, and reaches an asymptotic value in the central region of the fiber. 


\section{ACKNOWLEDGMENTS}

The authors thank Drs. E. Y. Sun and K. J. Kozaczek for reviewing the manuscript. Research sponsored by the U.S. Department of Energy, Division of Materials Sciences, Office of Basic Energy Sciences, under contract DE-AC05$840 R 21400$ with Lockheed Martin Energy Systems.

\section{REFERENCES}

1. A. G. Evans and R. M. Cannon, "Toughening of Brittle Solids by Martensitic Transformations," Acta Metall., 34 [5] 761-800 (1986).

2. P. F. Becher, C. H. Hsueh, P. Angelini and T. N. Tiegs, "Toughening Behavior in Whisker-Reinforced Ceramic Composites," J. Am. Ceram. Soc., 71 [12] 1050-61 (1988).

3. N. Hirosaki, Y. Akimune, and M. Mitomo, "Microstructure Characterization of Gas-Pressure-Sintered $\beta$-Silicon Nitride Containing Large $\beta$-Silicon Nitride Seeds," J. Am. Ceram. Soc., 77 [4] 1093-97 (1994).

4. J. D. Eshelby, "The Determination of the Elastic Field of an Ellipsoidal Inclusion, and Related Problems," Proc. Roy. Soc. London, A241, 376-96 (1957).

5. T. Mori and K. Tanaka, "Average Stress in Matrix and Average Elastic Energy of Materials with Misfitting Inclusions," Acta Metall., 21 [5] 571-74 (1973).

6. A. Abuhasan, C. Balasingh, and P. Predecki, "Residual Stresses in Alumina/Silicon Carbide (Whisker) Composites by X-Ray Diffraction," J. Am. Ceram. Soc., 73 [8] 2474-84 (1990).

7. S. Kumar and R. N. Singh, "Three-Dimensional Finite Element Modeling of Residual Thermal Stresses in Graphite/Aluminum Composites," Acta Metall. Mater., 43 [6] 2417-28 (1995).

8. C. H. Hsueh, "A Modified Analysis for Stress Transfer in Fibre-Reinforced Composites with Bonded Fibre Ends," J. Mater. Sci., 30, 219-24 (1995).

9. X. Yang and R. J. Young, "Determination of Residual Strains in CeramicFibre Reinforced Composites using Fluorescence Spectroscopy," Acta Metall. Mater., 43 [6] 2407-16 (1995).

10. Z. Li and R. C. Bradt, "Micromechanical Stresses in SiC-Reinforced $\mathrm{Al}_{2} \mathrm{O}_{3}$ Composites," J. Am. Ceram. Soc., 72 [1] 70-77 (1989).

11. C. H. Hsueh and P. F. Becher, "Residual Thermal Stresses in Ceramic Composites, Part I: with Ellipsoidal Inclusions," to be published.

12. C. H. Hsueh, "Sintering Behavior of Powder Compacts with Multiheterogeneities," J. Mater. Sci., 21 [6] $2067-72$ (1986).

13. C. H. Hsueh and P. F. Becher, "Thermal Expansion Coefficients of Unidirectional Fiber-reinforced Ceramics," J. Am. Ceram. Soc., 71 [10] C438-41 (1988).

14. H. L. Cox, "The Elasticity and Strength of Paper and Other Fibrous Materials," Brit. J. Appl. Phy., 3, 72-79 (1952).

15. C. H. Hsueh and P. F. Becher, "Residual Thermal Stresses in Ceramic Composites, Part II: with Short Fibers," to be published. 


\section{DISCLAIMER}

This report was prepared as an account of work sponsored by an agency of the United States Government. Neither the United States Government nor any agency thereof, nor any of their employees, makes any warranty, express or implied, or assumes any legal liability or responsibility for the accuracy, completeness, or usefulness of any information, apparatus, product, or process disclosed, or represents that its use would not infringe privately owned rights. Reference herein to any specific commercial product, process, or service by trade name, trademark, manufacturer, or otherwise does not necessarily constitute or imply its endorsement, recommendation, or favoring by the United States Government or any agency thereof. The views and opinions of authors expressed herein do not necessarily state or reflect those of the United States Government or any agency thereof. 